\title{
Amodal semantic representations depend on both anterior temporal lobes: Evidence from repetitive transcranial magnetic stimulation
}

\author{
Gorana Pobric $^{\mathrm{a}, *}$, Elizabeth Jefferies $^{\mathrm{b}}$, Matthew A. Lambon Ralph ${ }^{\mathrm{a}}$ \\ a Neuroscience and Aphasia Research Unit, School of Psychological Sciences, University of Manchester, UK \\ ${ }^{\mathrm{b}}$ Department of Psychology and York Neuroimaging Centre, University of York, UK
}

\section{A R T I C L E I N F O}

\section{Article history:}

Received 26 May 2009

Received in revised form

17 December 2009

Accepted 22 December 2009

Available online 28 December 2009

\section{Keywords:}

Semantic memory

Anterior temporal lobe

rTMS

Pictures

Words

\begin{abstract}
A B S T R A C T
The key question of how the brain codes the meaning of words and pictures is the focus of vigorous debate. Is there a "semantic hub" in the temporal poles where these different inputs converge to form amodal conceptual representations? Alternatively, are there distinct neural circuits that underpin our comprehension of pictures and words? Understanding words might be primarily left-lateralised, linked to other language areas, while semantic representation of pictures may be more bilateral. To elucidate this debate, we used offline, low-frequency, repetitive transcranial magnetic stimulation (rTMS) to disrupt neural processing temporarily in the left or right temporal poles. During the induced refractory period, participants made judgements of semantic association for verbal and pictorial stimuli. The efficiency of semantic processing was reduced by rTMS, yet a perceptual task of comparable difficulty was unaffected. rTMS applied to the left or right temporal poles disrupted semantic processing for words and pictures to the same degree, while rTMS delivered at a control site had no impact. The results confirm that both temporal poles form a critical substrate within the neural network that supports conceptual knowledge, regardless of modality.
\end{abstract}

(c) 2010 Elsevier Ltd. All rights reserved.

\section{Introduction}

Semantic memory, a type of declarative memory, consists of knowledge-based information, which is context independent and culturally shared (Tulving \& Schacter, 1990). It incorporates knowledge across multiple domains and allows us to understand the meanings of words, pictures, objects and faces (Patterson, Nestor, \& Rogers, 2007; Rogers \& McClelland, 2004; Rogers et al., 2004). As semantic memory stores our fundamental knowledge about the world, it is essential to functioning in our daily lives, communicating our thoughts, understanding the meaning of others and using the objects around us (Hart et al., 2007). Crucially, our semantic representations allow us to generalise knowledge appropriately from one exemplar to another (Lambon Ralph \& Patterson, 2008).

Because of its importance, the representation of semantic knowledge in the human brain is a matter of debate and controversy. Neuropsychological evidence comes from patients with semantic dementia (SD), who have a highly specific impairment

\footnotetext{
* Corresponding author at: Neuroscience and Aphasia Research Unit (NARU), School of Psychological Sciences (Zochonis Building), University of Manchester, Oxford Road, Manchester M13 9PL, UK. Tel.: +44 0161275 1967; fax: +44 01612752873

E-mail address: gorana.pobric@manchester.ac.uk (G. Pobric).
}

of semantic memory: they fail diverse semantic tasks even though other aspects of cognition and language, such as phonology, visual processing and decision-making remain intact (Hodges, Patterson, Oxbury, \& Funnell, 1992; Snowden, Goulding, \& Neary, 1989). The selective nature of the semantic impairment is coupled with a specific pattern of brain damage: SD patients have bilateral atrophy and hypometabolism in the anterior temporal lobes, maximal in the inferior and lateral aspects, and the extent of this atrophy correlates with the severity of the semantic impairment (Mummery et al., 2000; Nestor, Fryer, \& Hodges, 2006). These patient studies have given rise to the "semantic hub" hypothesis in which the anterior temporal lobes form a neural centre point where different inputs converge to form amodal conceptual representations (Patterson et al., 2007; Rogers et al., 2004; Rogers \& McClelland, 2004). This hypothesis differs from other theories suggesting that distinct neural circuits that underpin our comprehension of pictures and words (Martin, 2007). Whilst the brain damage in SD is remarkably circumscribed and consistent across patients, it is always possible that the semantic impairment actually results from pathology in regions beyond those maximally damaged. The atrophy and associated hypometabolism of SD is focused upon the anterior, polar aspects of the temporal lobes bilaterally with consistent and substantial grey matter loss in the polar and perirhinal cortices and the anterior fusiform gyri, bilaterally (Patterson et al., 2007). The simplest and most obvious hypothesis, therefore, is that these regions are critical for semantic memory (Lambon Ralph \& Patterson, 2008; Patterson 
et al., 2007; Rogers et al., 2004). Given that SD is a neurodegenerative condition, there is no absolute boundary to the damage. There is, therefore, always the possibility that sub-threshold damage or dysfunction due to invading pathology occurs elsewhere and it is this more subtle, widespread damage that is the root of the patients' semantic impairment (Martin, 2007). In addition, because SD is characterised by bilateral atrophy, it is not possible to investigate the contribution of left and right ATL in isolation. Therefore, the contributions of the ATL to semantic processing are not clearly defined on the basis of this neuropsychological evidence alone.

While the data arising from semantic dementia seem to implicate the temporal poles, bilaterally, in semantic representation, these areas are often overlooked or even disputed. Studies of various patient groups and functional neuroimaging in normal participants have consistently demonstrated a critical role of left prefrontal and temporoparietal regions in semantic cognition (Berthier, 1995; Devlin, Matthews, \& Rushworth, 2003; Thompson-Schill, D’Esposito, Aguirre, \& Farah, 1997). More importantly, imaging studies of semantic memory or comprehension rarely activate anterior temporal lobe regions but, in line with the aphasiological models, find activation in left temporoparietal and prefrontal regions (Martin, 2007). However, an influential PET study found evidence of anterior left middle temporal activation in associative-semantic task for both words and pictures (Vandenberghe, Price, Wise, Josephs, \& Frackowiak, 1996). The failure to find anterior temporal lobe activation in semantic tasks reflects, at least in part, various technical limitations (Visser et al., in press). The first is that a surprisingly large number of studies have used a restricted field-of-view (both PET and fMRI) and consequently have not sampled from the inferior aspects of the ATL. The second factor is that field inhomogeneities around air-filled cavities lead to signal drop out and distortions that are particularly pronounced in orbitofrontal cortex and the inferior and polar aspects of the temporal lobes (Devlin et al., 2000; Wise, 2003). Functional neuroimaging that utilises PET (which does not suffer from the same problems) does detect semantically related activation in the anterior temporal lobes, even when the same experiment conducted in fMRI does not (Devlin et al., 2000).

Finally, following unilateral resection of the temporal pole, epilepsy patients are not commonly reported to have semantic impairment or at least not to the same degree as SD patients (Hermann, Davies, Foley, \& Bell, 1999). Whilst this clinical comparison is an important one, the data from the TLE patients need to be treated with some caution given that these patients have longstanding epilepsy that might lead to functional re-organisation prior to surgery. Indeed there is already evidence that white matter connectivity and neurotransmitter function are significantly altered in this patient group (Powell et al., 2007).

Given these uncertainties over various aspects of patient and neuroimaging results, there is considerable debate in the literature about the putative role of different brain regions in semantic cognition, with strong advocates for the importance of one brain region over another (Hickok \& Poeppel, 2007; Martin, 2007; Patterson et al., 2007; Wise, 2003). An alternative approach is to use repetitive transcranial magnetic stimulation (rTMS) to disrupt processing briefly within the ATL of normal volunteers. A long train of low-frequency rTMS over a particular cortical field produces a temporary "virtual lesion" (Walsh \& Rushworth, 1999), impairing performance on specific tasks that rely on this area. A key advantage of rTMS is that it produces relatively small and reproducible temporary lesions. Moreover, several different "virtual lesions" can be compared in the same participant to examine the separate contributions of different areas, such as left and right ATL, that do not readily dissociate in neuropsychological investigations.

Various interpretations of the role of the ATL in semantic memory make different predictions for this study. The primary hypothesis, stemming from SD patient data, is that unified, amodal representations are underpinned by the ATL bilaterally. This follows from the fact that SD patients exhibit poor comprehension of items presented in every modality, including spoken and written words, pictures, environmental sounds, smells and touch (Bozeat, Lambon Ralph, Patterson, Garrard, \& Hodges, 2000; Coccia, Bartolini, Luzzi, Provinciali, \& Lambon Ralph, 2004; Luzzi et al., 2007). The marked semantic deficit is also apparent in production tasks, such as picture naming (Lambon Ralph, McClelland, Patterson, Galton, \& Hodges, 2001), verbal definitions (Lambon Ralph, Graham, Patterson, \& Hodges, 1999), object drawing (Bozeat et al., 2003) and object use (Bozeat, Lambon Ralph, Patterson, \& Hodges, 2002). The amodal nature of ATL processing is underlined by the strong item-specific consistency between different input and output modalities exhibited by SD patients (Bozeat et al., 2000; Gainotti, 2007).

A second possibility is that there are two separate ATL representational hubs in the left and right cerebral hemispheres. These hubs might have different specialisations. SD patients with more left- than right-sided atrophy $(L>R)$ have greater word-finding problems (anomia) for any given level of comprehension deficit (Lambon Ralph et al., 2001). Snowden, Thompson, and Neary (2004) demonstrated that $\mathrm{SD}$ patients with $\mathrm{L}>\mathrm{R}$ atrophy identified famous people better from faces than names, while $\mathrm{R}>\mathrm{L}$ patients showed the opposite pattern. One possibility, therefore, is that the left ATL is specialised for verbal semantic processing because language representations are left-lateralised: for example, using a connectionist model, Lambon Ralph et al. (2001) accounted for left-right asymmetries in picture naming in terms of stronger connections between left ATL and speech output processes in the left hemisphere. Extending this hypothesis, it is possible that the right temporal lobe might make a greater contribution to semantic processing for pictures if the perceptual information that interacts with semantic representations comes more strongly from right than left posterior regions (Gainotti, 2007), or if the preferential left hemisphere language connectivity induces a division of labour across the ATLs such that the right ATL defaults to nonverbal processing.

The third hypothesis is that only the left ATL plays a crucial role in semantic memory, with the right ATL not contributing in the same way. It is undisputed that the left hemisphere has a central role in the comprehension and production of spoken language (Wise, 2003). Furthermore, the importance of left anterior temporal cortex for language processing and semantic memory has been highlighted with PET (Devlin et al., 2000; Perani et al., 1999; Vandenberghe et al., 1996), intercranial recordings (Halgren et al., 1994) and MEG (Marinkovic et al., 2003). The corollary of this hypothesis is that although SD is a bilateral disease, the selective semantic impairment actually arises from damage to left ATL alone.

To distinguish among these hypotheses, we employed rTMS to induce a "virtual lesion" in left and right ATL in neurological intact participants. In a previous study, we found that this form of rTMS ( $1 \mathrm{~Hz}$ for $10 \mathrm{~min}$ ) applied to left temporal pole (TP) significantly slowed performance on both picture naming and a verbal comprehension task (synonym judgement), mimicking two of the core deficits observed in SD (Pobric, Jefferies, \& Lambon Ralph, 2007). These findings were extended by confirming that both temporal poles make a critical contribution to the neural network that supports verbal conceptual knowledge (Lambon Ralph, Pobric, \& Jefferies, 2009). In this study, we used rTMS to investigate the role of left and right ATL in a nonverbal semantic task for the first time. We directly compared semantic processing for the same concepts presented as words or pictures, yielding important new insights into the nature of the semantic representations formed in left and right ATL. The verbal condition also provides an important replication test for our previous studies which so far have only used one type of semantic task (synonym judgment). 


\section{Methods}

\subsection{Design}

A $3 \times 3 \times 2$ repeated-measures design was used, with site (left ATL vs. right ATL vs. occipital pole), task (word semantic association vs. picture semantic association vs. pattern matching) and TMS (no TMS vs. rTMS stimulation) as the 3 withinparticipant factors. The study utilised rTMS using the "virtual lesion" method in which the train of rTMS is delivered offline (without a concurrent behavioural task) and then behavioural performance is probed during the temporary refractory period and compared to performance on the same task outside this refractory window. In pilot studies, we found that rTMS and the associated novel experience, irrespective of site of stimulation, is highly alerting for participants. As a consequence there is a non-specific speeding of reaction times (on all tasks). Accordingly, the study was designed to deconfound order and the specific TMS effect. Half of the participants produced their "baseline", no-TMS data before rTMS was applied. The other half provided their baseline at least $30 \mathrm{~min}$ or more after the end of rTMS (by which time, our pilot studies indicate that no behavioural effect remains).

\subsection{Participants}

Ten, right-handed participants took part in the experiment (5 females; mean age $=21.5$ years, $S D=2.4$ ). All were native English speakers and strongly righthanded, yielding a laterality quotient of at least +90 on the Edinburgh Handedness Inventory (Oldfield, 1971). They were free from any history of neurological disease or mental illness and not on any medication. All had normal or corrected-to-normal vision. All gave written informed consent and the experiment was reviewed and approved by the local ethics board. Participants were reimbursed for their participation.

\subsection{Stimuli}

One hundred and twenty semantic association trials were created by combining the Pyramids and Palm Trees test (PPT) (Howard \& Patterson, 1992) and an abridged version of the Camel and Cactus Test (CCT) (Bozeat et al., 2000). The alteration of the Camel and Cactus test consisted of reducing the number of choice items to two (instead of four same category items). We also added 10 items from the Location Refractory Test. This gave us the number of stimuli needed for a TMS experiment. Both picture and word versions of the tests were used. Pattern images were created by scrambling the pictures and words used in the semantic association task. All patterns were created in Java Runtime Environment (www.SunMicrosystems.com) by scrambling pictures into 80 pieces and rearranging them in a random fashion. Words were scrambled into 15 pieces and rearranged randomly.

\subsection{Task and procedure}

A PC running E-Prime software (Psychology Software Tools Inc., Pittsburgh, USA) allowed the presentation of stimuli and recording of the responses. The participants sat $57 \mathrm{~cm}$ in front of a $15 \mathrm{in}$. monitor.

Participants performed two semantic association tasks (pictures and words) and two pattern matching tasks (scrambled pictures and scrambled words) per experimental session (one within and one outside the rTMS induced refractory period-see above). In a single experimental session, participants saw the entire PPT and CCT batteries. Half of the battery was presented as pictures, the other half as words This order was counterbalanced across stimulation sites. The experiment began with a practice block of 10 trials for each stimulus set. Experimental trials were presented in a random order in 4 blocks of 30 trials (pictures, words, scrambled pictures and scrambled words-120 trials in total). All blocks were randomised across participants. A fixation point lasting $500 \mathrm{~ms}$ appeared on the screen to signal the start of each trial. Stimuli (pictures, words, scrambled images) were presented until response or for a maximum of $3000 \mathrm{~ms}$ followed by a blank screen interval of $500 \mathrm{~ms}$. Participants were shown a sample picture (word), with two choice pictures (words) below. The task was simply to indicate which of the two choice stimuli was more closely related to the sample, shown at the top of the screen (see Fig. 1). In the pattern matching task, participants were presented with a scrambled image (picture or word) and they had to indicate which of two choice patterns was a vertically flipped mirror-image of the sample (see Fig. 1). Participants indicated their choice by pressing with the right hand one of two designated keys on a keyboard that corresponded with the location of their selected item as fast as possible. After $10 \mathrm{~min}$ of rTMS stimulation, participants performed another 4 blocks of 30 trials (a post rTMS condition). As noted above, whether the non-TMS session was conducted before or after (at least $30 \mathrm{~min}$ ) the TMS session was counterbalanced across participants to deconfound TMS and order effects.

\subsection{TMS}

A MagStim Rapid2 (Magstim Co., Whitland, UK) stimulator with two external boosters was used (maximum output approximately $2.2 \mathrm{~T}$ ). Magnetic stimulation was applied using a 70-mm figure-of-eight coil. The double wire windings which make up the figure-of-eight coil carry two alternating electrical currents which con- verge at the point where the two coils meet (at the centre of the figure-of-eight). A quite focal electrical current can then be induced in the cortex via magnetic conduc tion from this central point which undergoes minimal attenuation by the intervening soft tissue and bone (Jalinous, 1995). Previous studies have demonstrated that magnetic stimulation using this type of coil can produce functionally dissociable effects when moving the coil by $5-10 \mathrm{~mm}$ across the scalp (Brasil-Neto, McShane, Fuhr, Hallett, \& Cohen, 1992)

\subsection{Anatomical MRI acquisition}

3D high-resolution T1-weighted anatomical images were acquired for all participants using a 3T Philips MR Achieva scanner (Philips Electronics, The Netherlands) MRI scanning parameters included an in-plane resolution of $1 \mathrm{~mm}$ and a slice thick ness of $1.8 \mathrm{~mm}$ with an acquisition matrix of $256 \times 256$ voxels. The number of contiguous axial slices acquired varied $(\max =240)$ depending on the size of the subject's head, as full head coverage was required for accurate co-registration of the image to the participants scalp. These high-resolution T1-weighted images enabled reconstruction of the fine individual cortex folding which was used as anatomical landmarks for the TMS targets.

\subsection{Selection of TMS site}

The structural T1-weighted MRI scans were co-registered with the participant's scalp using MRIreg (www.mricro.com/mrireg.html). Immediately prior to the TMS session, scalp coordinates were measured using an Ascension Minibird (www.ascension-tech.com) magnetic tracking system. A series of scalp landmarks were identified for co-registration within the MRI and Minibird coordinates. Once this calibration was complete, the two frames of reference were co-registered using least squares linear estimation. This allowed us to compare the position of the Minibird on the scalp to the underlying cortical surface. From the tip of the temporal pole we measured $10 \mathrm{~mm}$ posterior along the middle temporal gyrus. This point was used in each participant as an anatomical landmark for the ATL. The location of the ATL was identified on each participant and the scalp location directly above this site was marked with a permanent marker. The left MNI coordinates for the ATL in standard space were $(-53,4,-32)$. The same procedure was used for locating the right ATL, which corresponded to average MNI coordinates of ( 52 $2,-28$ ) in standard space. A middle occipital stimulation site (occipital pole) was also employed as a site to control for possible non-specific visual effects and also for general arousal effects of TMS induced by somatosensory and acoustic artifacts. According to the international 10-20 electrode system, this site corresponds to the Oz location (Fig. 2)

\subsection{Stimulation parameter}

Individual motor threshold (MT) was determined for every participant. Motor threshold was defined as a minimal intensity of stimulation capable of inducing motor evoked potentials in the contralateral FDI muscle greater than $50 \mu \mathrm{V}$ peakto-peak amplitude in at least 6 out of 10 trials (Rossini et al., 1994) at the optima scalp position. Repetitive pulse TMS (rTMS) was delivered offline for $10 \mathrm{~min}$ at $1-\mathrm{Hz}$ ( $600 \mathrm{~s}$ at $120 \%$ motor threshold level) applied to the left and right temporal pole, and occipital pole. The coil was securely held against the left/right temple, centred over the site to be stimulated. This TMS protocol has been shown to produce behavioural effects that last for several minutes after stimulation (Hilgetag, Theoret, \& PascualLeone, 2001; Kosslyn et al., 1999). For the occipital stimulation the maximal induced current flowed downward with the coil handle pointing upwards. The average MT was $54 \%$ of the maximal stimulator output and the average stimulation intensity during rTMS was $65 \%$.

\subsection{Methodological considerations}

An advantage of low-frequency rTMS is that rTMS modulates the level of excitability of a given cortical area beyond the duration of the rTMS train itself (Knecht et al., 2002). In the present design, behaviour was evaluated before and after rTMS. Therefore, a non-specific disruption of performance due to discomfort, noise, muscle twitches and intersensory facilitation associated with rTMS during the task was avoided. rTMS has a considerable alerting effect irrespective of task or location of stimulation and thus has a generic speeding effect on decision times in cognitive tasks. Accordingly, we deconfounded the effects of TMS and order in the experiments. Particular care was taken in the placing of the TP coil because TMS here is more uncomfortable than over occipital or parietal areas. We manipulated coil orientation (a major factor in the nature of the contraction of facial/neck mus cles) to find an orientation that minimized the discomfort to a subjective equivalent to that of the stimulation over other sites. As detailed above, we also used a pattern recognition task as a control to ensure that neither non-specific effects of the rTMS procedure nor task difficulty could explain the observed results. 

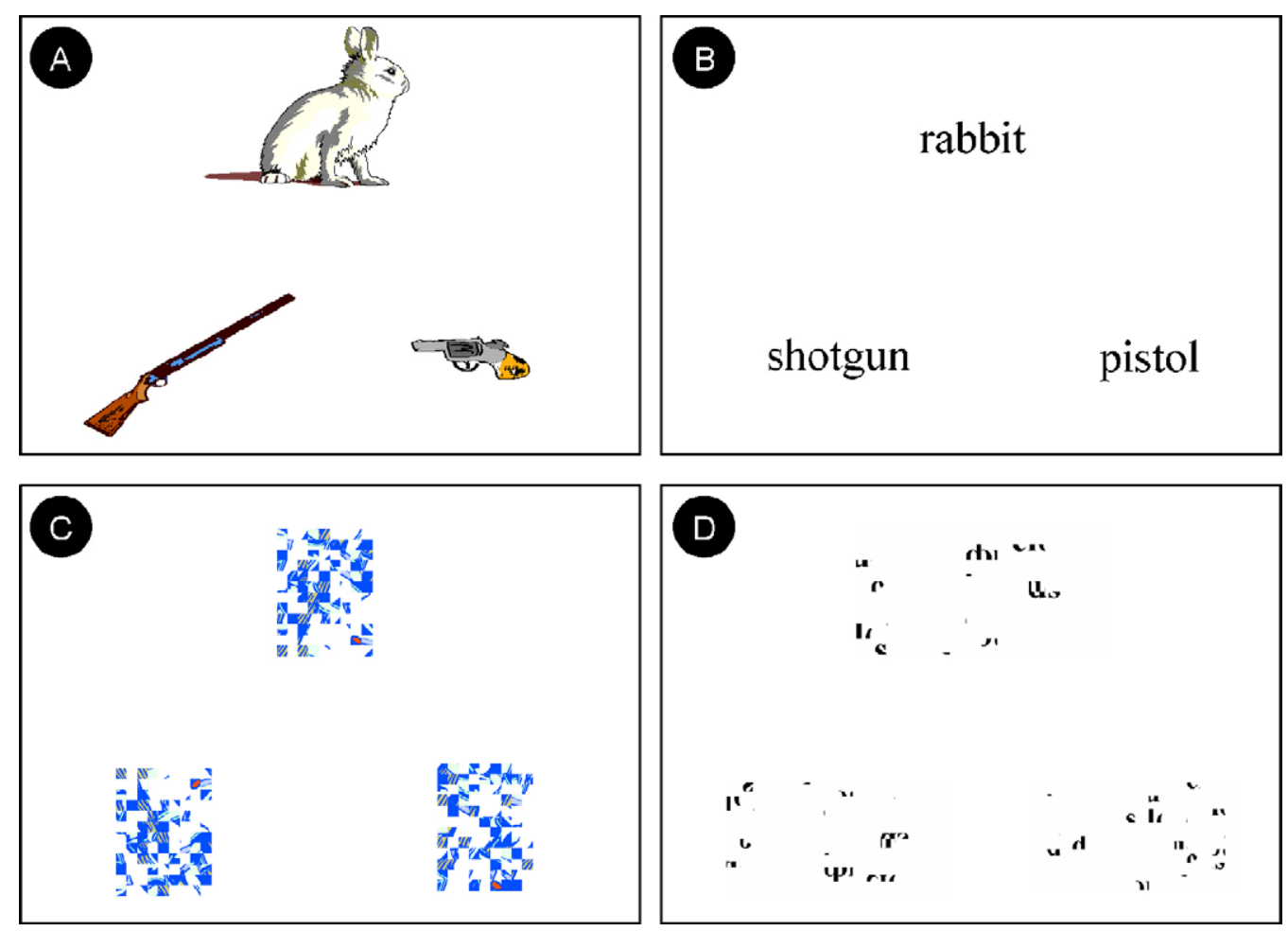

Fig. 1. Example stimuli for the semantic association task using pictures (A) and words (B) and for the mirror-image pattern matching task using scrambled pictures (C) and scrambled words (D).

\section{Results}

\subsection{Response times}

The response times (RT) for all participants and all conditions were submitted to a repeated-measures ANOVA with three withinsubjects factors: stimulus (pictures, words and scrambled images - patterns), site (left ATL, right ATL and Oz) and TMS (rTMS vs. no TMS). Since no difference was observed between scrambled pictures and scrambled words $(p>.4)$, these were collapsed into a single control condition (scrambled patterns) in the main analyses. There were no significant main effects of either stimulus $[F=1.39, \mathrm{df}=2,18, p=\mathrm{n} . \mathrm{s}$. $]$ or stimulation site $[F=2.21, \mathrm{df}=2,18$, $p=n . s$.]; however, the main effect of TMS approached significance $[F=4.53, \mathrm{df}=1,9, p=.06]$. There was a significant interaction between stimulus type and site of stimulation $[F=11.43, \mathrm{df}=2,18$, $p<.001]$ and between site and TMS $[F=7.89, \mathrm{df}=2,18, p=.003]$. Most importantly, there was a significant three-way interaction between stimulation site, stimulus type and TMS $[F=3.72, \mathrm{df}=4,36$, $p=.04]$. Because of the significant three-way interaction, we split the analysis along the site dimension. We performed three separate $3 \times 2$ ANOVAs for each stimulated site (Oz, left ATL and right ATL). For occipital pole, we did not observe a significant main effects of stimulus type $[F=1.21, \mathrm{df}=2,18, p=$ n.s. $]$, but the main effect of TMS was significant $[F=6.15, \mathrm{df}=1,9, p=.035]$ contributing to a general speeding effect caused by practice effects (see Fig. 3 ). The interaction between stimulus type and TMS was not significant $[F=.73$, $\mathrm{df}=2,18, p=n . s$.]. For left ATL we did not observe a significant main effects of stimulus type $[F=1.136, \mathrm{df}=2,18, p=\mathrm{n} . \mathrm{s}$.], however the main effect of TMS approached significance $[F=4.42, \mathrm{df}=1,9$, $p=.065$.]. Also, there was a significant interaction between stimulus type and TMS $[F=14.82, \mathrm{df}=2,18, p<.001]$. For right ATL we did not observe a significant main effects of stimulus type $[F=0.69$, $\mathrm{df}=2,18, p=n . s$.], however the main effect of TMS was significant $[F=11.49, \mathrm{df}=1,9, p=.008]$. More importantly, there was a significant interaction between stimulus type and TMS $[F=4.53, \mathrm{df}=2,18$,

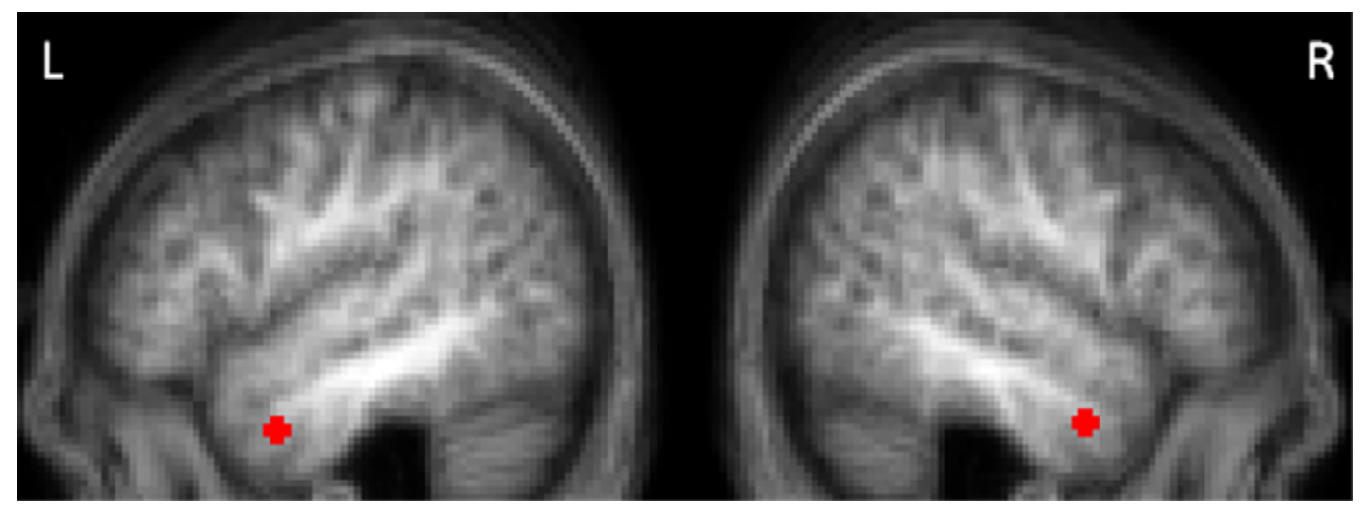

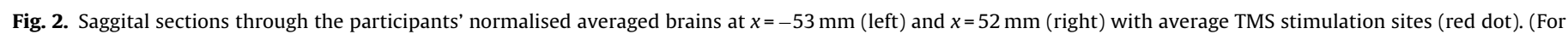
interpretation of the references to colour in this figure legend, the reader is referred to the web version of the article.) 


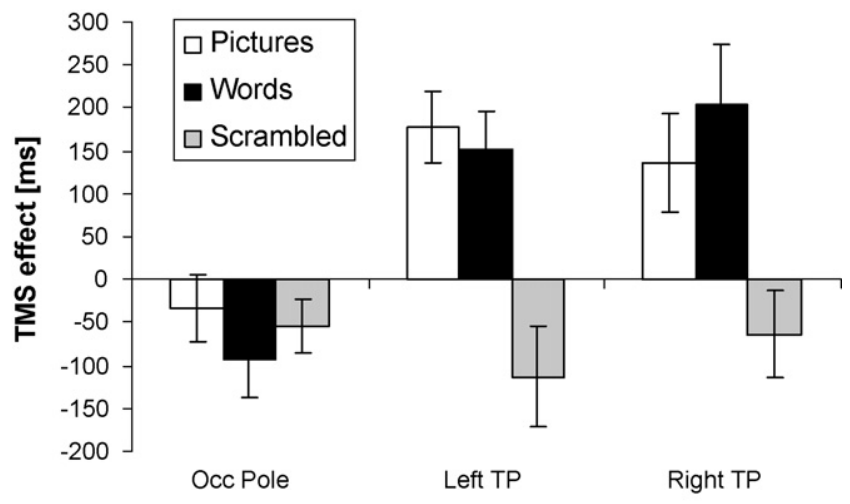

TMS Site

Fig. 3. The TMS effect on semantic association and pattern judgement tasks. Each bar represents the TMS effect in terms of the reaction time (RT) change caused by TMS. For example, the white bar above the left TP site indicates that rTMS over left TP caused a RT delay of $177 \mathrm{~ms}$ for picture association judgments, when compared to the no-TMS condition. Negative figures indicate RTs on TMS trials were faster than no-TMS trials. Error bars indicate SEM adjusted to reflect the between-condition variance used in repeated-measure designs (Loftus \& Masson, 1994).

$p=.025]$. Planned comparisons $t$-tests were used to compare performance for each stimulus set (pictures, words and scrambled patterns) with and without TMS at each site showing significant interactions (see Fig. 3). After controlling for False Discovery Rate (Benjamini \& Hochberg, 1995) the following planned comparisons were significant: stimulation of left ATL significantly slowed performance for both the picture association task $[t(9)=4.3, p=.002]$ and the word association task $(t(9)=3.47, p=.007]$. Right ATL stimulation also reliably slowed both picture association $[t(9)=2.34$, $p=.04]$ and word association performance $[t(9)=2.92, p=.02]$ (see Fig. 4). TMS did not have any significant effects on the control task (scrambled images). In order to rule out the possibility that the difference between left and right ATL stimulation for semantic tasks was due to lack of power, we computed the effect sizes for the four significant TMS effects: Pictures Left $=1.36$; Pictures Right $=.74$; Words Left $=1.10$; Words Right $=.92$, respectively. Regarding the difference between stimulation of left and right ATL, the effect sizes

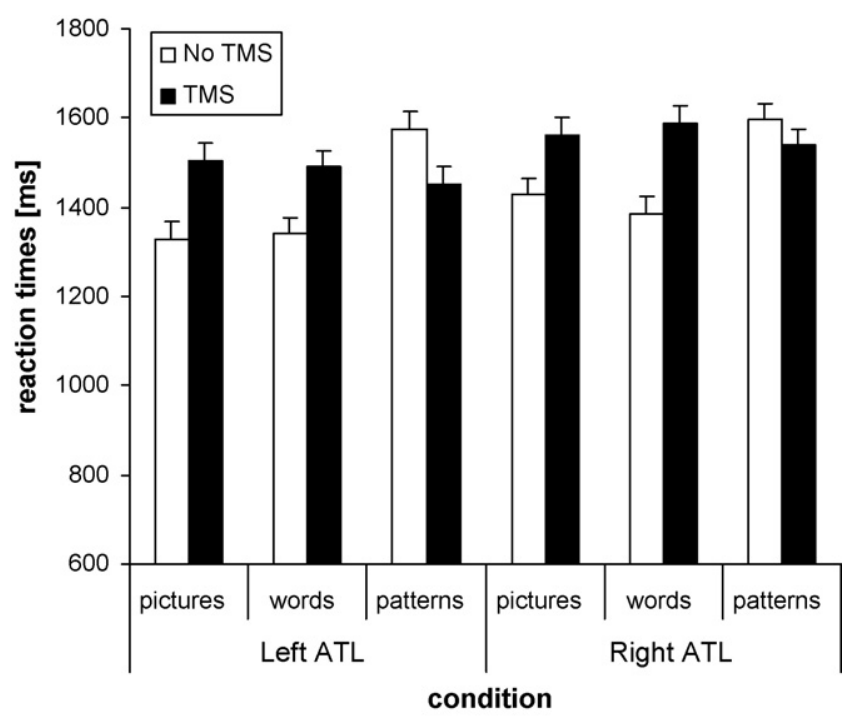

Fig. 4. Semantic association and pattern judgment tasks employing pictures and words. Each bar represents the mean decision time alongside the corresponding standard error adjusted for within subject comparisons (Loftus \& Masson, 1994) for each condition. were much smaller: for the pictures the effect size was only .18 and for the words it was .21. Moreover, the differences between left and right stimulation went in opposite directions for words and pictures, yielding an overall effect size for the difference between left and right TMS stimulation for semantic tasks of only .03. It seems unlikely, therefore, that the lack of a difference between left and right ATL stimulation for semantic tasks was due to lack of power.

\subsection{Error analyses}

The error rate was examined in a repeated-measures ANOVA with stimulus (pictures, words and scrambled pictures), site (left ATL, right ATL and Oz) and TMS as factors. The main effect of stimulus $[F=4.08, \mathrm{df}=2,18, p=.06]$ approached significance. There were no significant main effects of either stimulation site $[F=0.28$, $\mathrm{df}=2,18, p=$ n.s.]; or TMS [ $F=0.16, \mathrm{df}=1,9, p=$ n.s. $]$. There were no significant interactions between stimulus type and site of stimulation $[F=0.43, \mathrm{df}=2,18, p=$ n.s.], nor between site and TMS $[F=0.71$, $\mathrm{df}=2,18, p=n$.s.]. Also the three-way interaction between stimulation site, stimulus type and TMS $[F=0.68, \mathrm{df}=4,36, p=n$.s. $]$ was not significant. None of the planned comparison $t$-tests that contrasted error rates for each stimulus set (pictures, words and scrambled images) with and without TMS at each site were significant [all $p s>.2]$.

\section{General discussion}

The aims of this study were to demonstrate that the ATL regions are critically important in amodal representations of conceptual knowledge and, furthermore, to assess the contribution of the left and right ATL to this critical form of knowledge. When rTMS was applied to left or right ATL, neurologically normal participants exhibited the same degree of slowing on tasks requiring judgements of semantic association. The slowing effect of rTMS is fairly consistent across participants. For all four semantic effects (Pictures Left; Pictures Right; Words Left; Words Right) eight out of ten participants slowed down after rTMS. Moreover, nine of the ten participants slowed down for at least three conditions. This significant slowing was true irrespective of whether the stimuli were nonverbal (pictures) or verbal (written names) but there was no effect on equally demanding perceptual tasks. These findings are incompatible with the hypothesis that only the left ATL underpins semantic memory (even for verbal semantic tasks), because participants were impaired at both word and picture semantic tasks when the right ATL was stimulated. The results are also inconsistent with the idea of two differentially specialised semantic hubs in left and right ATL, because rTMS of these two regions produced equal slowing on word and picture tasks. Instead, we have demonstrated - for the first time - that the integrity of both left and right ATL is critical for processing the meanings of both words and pictures.

Of course, we cannot conclude that there are no subtle, graded differences in processing across the two hemispheres (which this study might have failed to detect, i.e., a type II error) but we can conclude that both hemispheres are involved in both word and picture versions of the task. In addition, there is growing evidence that TMS can generate remote effects on neural processing in interconnected regions (Ruff, Driver, \& Bestmann, 2009). In the current study, therefore, it is possible that stimulating the left ATL may have induced inhibition or alternatively compensatory excitation to the contralateral side (right ATL). If correct then this might weaken our ability to detect subtle differences between the hemispheres but by stimulating both sites we have confirmed our principal observation from SD patients that both left and right ATL are part of the functional network that supports semantic cognition (Lambon Ralph et al., 2001). Future studies combining fMRI and TMS will be 
able provide valuable information on how reduced excitability or compensatory changes in one brain area can affect more extended brain networks.

Evidence in favour of a single amodal hub in the ATL comes from studies of SD patients with circumscribed bilateral ATL atrophy. SD patients show comprehension deficits across the full range of input modalities (Bozeat et al., 2000; Luzzi et al., 2007) and have significant expressive difficulties in both verbal tasks (e.g., naming and speaking) (Lambon Ralph et al., 2001) and nonverbal domains (e.g., picture drawing and object use) (Coccia et al., 2004; Bozeat et al., $2002,2003)$. These findings suggest that the ATL extract an amodal semantic representation from a distillation of information available in different input and output codes. A similar view has been proposed by Damasio and colleagues in 'convergence zone' model of semantic memory (Damasio, 1989; Damasio, Grabowski, Tranel, Hichwa, \& Damasio, 1996; Tranel, Damasio, \& Damasio, 1997). It is proposed that certain regions of the brain serve as "convergence zones," by reactivating these distributed networks and serving to "bind" sensory and motor input to allow us to form conceptual impressions of our inner and outer worlds (Tranel et al., 1997). The convergence zones hypothesis differs from the amodal hub theory in various ways: (a) it proposes multiple specialised convergence regions, (b) it also suggests that convergence zones become differentially important for representing different semantic categories, and (c) at least as currently described, the convergence zones act as a simple feature linking device, whereas the ATL hub hypothesis includes an additional re-representation computation that is critical for semantically based generalisations (Patterson et al., 2007; Lambon Ralph \& Patterson, 2008).

From a neuroanatomical perspective, the ATL are an ideal substrate for forming amodal semantic representations as they are highly connected with many other modality-specific association and motor cortices in posterior temporal parietal and frontal regions (Gloor, 1997). Moreover, the amodal hypothesis has been implemented in computational models incorporating a central semantic "hub" that receives inputs from both verbal and visual systems (Plaut, 2002; Rogers \& McClelland, 2004; Rogers et al., 2004). Units within this "hub" allow the model to extract highorder, amodal representations about concepts. As a result, these representations are not dominated by similarities in any individual modality but instead reflect semantic relationships apparent across all of the modality-specific representations taken together. This neural implementation not only permits the translation of information between different sensory, verbal and motor modalities but, perhaps even more importantly, licenses correct semantic generalization across exemplars and concepts (Lambon Ralph \& Patterson, 2008). This means that although specific features of knowledge are represented in a widely distributed network of brain areas (Martin, 2007), including many regions beyond ATL, the ability to receive information in one modality and express it in another, to generalize across conceptually similar objects and to differentiate between superficially similar entities are quintessentially semantic abilities that depend on ATL (Lambon Ralph \& Patterson, 2008; Patterson et al., 2007).

The results of the present rTMS study decisively affirm this claim by showing that the temporal poles in both cerebral hemispheres make a necessary contribution to semantic tasks irrespective of input modality: pictures or words. They also allow us to reject the hypothesis that there is an absolute verbal-nonverbal distinction between left and right ATL. There may be alternative explanations of the apparent distinction between left and right ATL reported in two previous SD studies (Lambon Ralph et al., 2001; Snowden et al., 2004). Bilateral ATL may function as a single system but modality differences between the two cerebral hemispheres could still arise through differential patterns of connectivity to verbal and nonverbal inputs (Lambon Ralph et al., 2001).
Future studies utilising rTMS will be able to explore whether there is a graded specialisation in difference subregions of the ATL-perhaps reflecting differential patterns of white matter connectivity, as suggested by some functional neuroimaging studies and in some connectionist models (Plaut, 2002). In any event, our current findings confirm the importance of bilateral ATL for amodal semantic knowledge in neurologically intact individuals for the first time.

\section{Acknowledgements}

The study was supported by an RCUK fellowship, a Wellcome project grant (078734/Z/05/Z) and an MRC programme grant (G0501632).

\section{References}

Benjamini, Y., \& Hochberg, Y. (1995). Controlling the false discovery rate: A practical and powerful approach to multiple testing. Journal of the Royal Statistical Society Series B, 57, 289-300.

Berthier, M. L. (1995). Transcortical sensory aphasia-Dissociation between naming and comprehension. Aphasiology, 9, 431-451.

Bozeat, S., Lambon Ralph, M. A., Patterson, K., Garrard, P., \& Hodges, J. R. (2000). Non-verbal semantic impairment in semantic dementia. Neuropsychologia, 38 , 1207-1215.

Bozeat, S., Lambon Ralph, M. A., Graham, K. S., Patterson, K., Wilkin, H., \& Rowl, J. (2003). A duck with four legs: Investigating the structure of conceptual knowledge using picture drawing in semantic dementia. Cognitive Neuropsychology, $20,27-47$.

Bozeat, S., Lambon Ralph, M. A., Patterson, K., \& Hodges, J. R. (2002). When objects lose their meaning: What happens to their use? Cognitive Affective and Behavioural Neuroscience, 2.

Brasil-Neto, J. P., McShane, L. M., Fuhr, P., Hallett, M., \& Cohen, L. G. (1992). Topographic mapping of the human motor cortex with magnetic stimulation: Factors affecting accuracy and reproducibility. Electroencephalography and Clinical Neurophysiology, 85, 9-16.

Coccia, M., Bartolini, M., Luzzi, S., Provinciali, L., \& Lambon Ralph, M. A. (2004). Semantic memory is an amodal, dynamic system: Evidence from the interaction of naming and object use in semantic dementia. Cognitive Neuropsychology, $21,513-527$

Damasio, A. R. (1989). The brain binds entities and events by multiregional activation from convergence zones. Neural Computation, 1, 123-132.

Damasio, H., Grabowski, T. J., Tranel, D., Hichwa, R. D., \& Damasio, A. R. (1996). A neural basis for lexical retrieval. Nature, 380, 499-505.

Devlin, J. T., Russell, R. P., Davis, M. H., Price, C. J., Wilson, J., \& Moss, H. E. (2000). Susceptibility-induced loss of signal: Comparing PET and fMRI on a semantic task. Neuroimage, 11, 589-600.

Devlin, J. T., Matthews, P. M., \& Rushworth, M. F. S. (2003). Semantic processing in the left inferior prefrontal cortex: A combined functional magnetic resonance imaging and transcranial magnetic stimulation study. Journal of Cognitive Neuroscience, $15,71-84$

Gainotti, G. (2007). Different patterns of famous people recognition disorders in patients with right and left anterior temporal lesions: A systematic review. Neuropsychologia, 45, 1591-1660.

Gloor, P. (1997). The temporal lobe and the limbic system. Oxford: Oxford University Press.

Halgren, E., Baudena, P., Heit, G., Clarke, J. M., Marinkovic, K., \& Calrke, M. (1994). Spatio-temporal stages in face and word processing. I. Depth-recorded potentials in the human occipital, temporal and parietal lobes. Journal of Physiology (Paris), 88, 1-50.

Hart, J., Jr., Anand, R., Zoccoli, S., Maguire, M., Gamino, J., Tillman, G., et al. (2007). Neural substrates of semantic memory. International Journal of Clinical Neuropsychological Society, 13, 865-880.

Hermann, B., Davies, K., Foley, K., \& Bell, B. (1999). Visual confrontation naming outcome after standard left anterior temporal lobectomy with sparing versus resection of the superior temporal gyrus: A randomized prospective clinical trial. Epilepsia, 40, 1070-1076.

Hickok, G., \& Poeppel, D. (2007). Opinion-The cortical organization of speech processing. Nature Reviews Neuroscience, 8, 393-402.

Hilgetag, C. C., Theoret, H., \& Pascual-Leone, A. (2001). Enhanced visual spatial attention ipsilateral to rTMS-induced 'virtual lesions' of human parietal cortex. Nature Neuroscience, 4, 953-957.

Hodges, J. R., Patterson, K., Oxbury, S., \& Funnell, E. (1992). Semantic dementia: Progressive fluent aphasia with temporal lobe atrophy. Brain, 115, 1783-1806.

Howard, D., \& Patterson, K. (1992). Pyramids and palm trees: A test of semantic access from pictures and words. Bury St. Edmunds, UK: Thames Valley Test Co.

Jalinous, R. (1995). Guide to magnetic stimulation. Wales, Magstim: Whitland.

Knecht, S., Floel, A., Drager, B., Breitenstein, C., Sommer, J., Henningsen, H., et al. (2002). Degree of language lateralization determines susceptibility to unilateral brain lesions. Nature Neuroscience, 5, 695-699. 
Kosslyn, S. M., Pascual-Leone, A., Felician, O., Camposano, S., Keenan, J. P., Thompson, W. L., et al. (1999). The role of Area 17 in visual imagery: Convergent evidence from PET and rTMS. Science, 284, 167-170.

Lambon Ralph, M. A., \& Patterson, K. (2008). Generalisation and differentiation in semantic memory: Insights from semantic dementia. Annals of the New York Academy of Sciences, 1124, 61-76.

Lambon Ralph, M. A., McClelland, J. L., Patterson, K., Galton, C. J., \& Hodges, J. R. (2001). No right to speak? The relationship between object naming and semantic impairment: Neuropsychological evidence and a computational model. Journal of Cognitive Neuroscience, 13, 341-356.

Lambon Ralph, M. A., Graham, K. S., Patterson, K., \& Hodges, J. R. (1999). Is a picture worth a thousand words? Evidence from concept definitions by patients with semantic dementia. Brain and Language, 70, 309-335.

Lambon Ralph, M. A., Pobric, G., \& Jefferies, E. (2009). Conceptual knowledge is underpinned by temporal pole bilaterally: Convergent evidence from rTMS. Cerebral Cortex, 19, 832-838.

Loftus, G. R., \& Masson, M. E. J. (1994). Using confidence intervals in within-subject designs. Psychonomic Bulletin \& Review, 1, 476-490.

Luzzi, S., Snowden, J. S., Neary, D., Coccia, M., Provinciali, L., \& Lambon Ralph, M. A. (2007). Distinct patterns of olfactory impairment in Alzheimer's disease, semantic dementia, frontotemporal dementia, and corticobasal degeneration. Neuropsychologia, 45, 1823-1831.

Marinkovic, K., Dhond, R. P., Dale, A. M., Glessner, M., Carr, V., \& Halgren, E. (2003). Spatiotemporal dynamics of modality-specific and supramodal word processing. Neuron, 38, 487-497.

Martin, A. (2007). The representation of object concepts in the brain. Annual Review of Psychology, 58, 25-45.

Mummery, C. J., Patterson, K., Price, C. J., Ashburner, J., Frackowiak, R. S. J., \& Hodges, J. R. (2000). A voxel based morphometry study of semantic dementia: The relation of temporal lobe atrophy to cognitive deficit. Annals of Neurology, 47, 36-45.

Nestor, P. J., Fryer, T. D., \& Hodges, J. R. (2006). Declarative memory impairments in Alzheimer's disease and semantic dementia. Neuroimage, 30, 1010-1020.

Oldfield, R. C. (1971). The assessment and analysis of handedness: The Edinburgh inventory. Neuropsychologia, 9, 97-113.

Patterson, K., Nestor, P. J., \& Rogers, T. T. (2007). Where do you know what you know? The representation of semantic knowledge in the human brain. Nature Reviews Neuroscience, 8, 976-987.

Perani, D., Cappa, S. F., Schnur, T., Tettamanti, M., Collina, S., Rosa, M., et al. (1999). The neural correlates of verb and noun processing. A PET study. Brain, 122, 2337-2344

Plaut, D. C. (2002). Graded modality-specific specialization in semantics: A computational account of optic aphasia. Cognitive Neuropsychology, 19, 603-639.
Pobric, G., Jefferies, E., \& Lambon Ralph, M. A. (2007). Anterior temporal lobes mediate semantic representation: Mimicking semantic dementia by using rTMS in normal participants. Proceedings of the National Academy of Sciences, 104, 20137-20141.

Powell, H. W. R., Parker, G. J. M., Alexander, D. C., Symms, M. R., Boulby, P. A., WheelerKingshott, C. A. M., et al. (2007). Abnormalities of language networks in temporal lobe epilepsy. Neuroimage, 36, 209-221.

Rogers, T. T., Lambon Ralph, M. A., Garrard, P., Bozeat, S., McClelland, J. L., \& Hodges, J. R. (2004). The structure and deterioration of semantic memory: A neuropsychological and computational investigation. Psychological Review, 111, 205-235.

Rogers, T. T., \& McClelland, J. L. (2004). Semantic cognition: A parallel distributed processing approach. Cambridge, MA: The MIT Press.

Rossini, P. M., Barker, A. T., Berardelli, A., Caramia, M. D., Caruso, G., Cracco, R. Q., et al. (1994). Non-invasive electrical and magnetic stimulation of the brain, spina cord and roots: Basic principles and procedures for routine clinical application. Report of an IFCN committee. Electroencephalography and Clinical Neurophysiology, 91, 79-92.

Ruff, C. C., Driver, J., \& Bestmann, S. (2009). Combining TMS and fMRI: From 'virtual lesions' to functional-network accounts of cognition. Cortex, 45, 1043-1049.

Snowden, J. S., Thompson, J. C., \& Neary, D. (2004). Knowledge of famous faces and names in semantic dementia. Brain, 127, 860-872.

Snowden, J. S., Goulding, P. J., \& Neary, D. (1989). Semantic dementia: A form of circumscribed cerebral atrophy. Behavioural Neurology, 2, 167-182.

Thompson-Schill, S. L., D’Esposito, M., Aguirre, G. K., \& Farah, M. J. (1997). Role of left inferior prefrontal cortex in retrieval of semantic knowledge: A reevaluation. Proceedings of the National Academy of Sciences, 94, 14792-14797.

Tranel, D., Damasio, H., \& Damasio, A. R. (1997). A neural basis for the retrieval of conceptual knowledge. Neuropsychologia, 35, 1319-11327.

Tulving, E., \& Schacter, D. L. (1990). Priming and human memory systems. Science, 247, 301-306.

Vandenberghe, R., Price, C., Wise, R., Josephs, O., \& Frackowiak, R. S. J. (1996) Functional-anatomy of a common semantic system for words and pictures. Nature, 383, 254-256.

Visser, M., Jefferies, B., \& Lambon Ralph, M. A. (in press) Semantic processing in the anterior temporal lobes: A meta-analysis of the functional neuroimaging literature. Journal of Cognitive Neuroscience. Epub ahead of print, July 7

Walsh, V., \& Rushworth, M. (1999). A primer of magnetic stimulation as a tool for neuropsychology. Neuropsychologia, 37, 125-135.

Wise, R. (2003). Language systems in normal and aphasic human subjects: Functional imaging studies and inferences from animal studies. British Medical Bulletin, 65, 95-119. 